\title{
Fatores de risco da automedicação em gestantes: um estudo sobre os Anti- inflamatórios não esteroides
}

\author{
Risk factors of self-medication in pregnant women: a study of non-steroidal Anti-inflammatory
}

drugs

Factores de riesgo para la automedicación en mujeres embarazadas: un estudio de fármacos Antiinflamatorios no esteroides

\begin{abstract}
Resumo
Objetivo: Analisar os riscos potenciais às Gestantes e Fetos, causados pela automedicação de anti-inflamatórios não esteroides. Métodos: A proposta de metodologia para esta pesquisa segue uma estratégia de revisão de literatura, pesquisa exploratória e descritiva, cuja coleta fora realizada em diferentes bases de dados, tais como Scientific Electronic Library Online (SCIELO), registros tanto da Biblioteca Virtual em Saúde (BVS), quanto registros da Medical Literature Analysis and Retrievel System Online (MEDLINE/PUBMED). A busca se deu através do uso dos descritores “AUTOMEDICAÇÃO”; “GESTANTES”, “AINES” e "RISCOS”. Resultados: Percebeu-se que a utilização de medicamentos durante a gravidez pela prática da automedicação com Ibuprofeno 9 (27\%), Dipirona 6 (18\%), Bromoprida 2 (6\%), Paracetamol 13 (40\%), Dimenidrinato 3 (9\%), sendo 31 (94\%) em forma de comprimidos foi relatada por $27(33,75 \%)$ gestantes, e $3(11,1 \%)$ que apresentaram queixas de uso como cefaleia, êmese e náuseas e a indicação foi relatada por $2(6 \%)$ gestantes pela a mãe e as demais por conta própria. Conclusão: Após a análise de diversos artigos científicos, o que fica bem explicito é que, o uso de anti-inflamatórios não esteroidais no início da gravidez não demonstrou ser um fator de risco, porém, após o primeiro trimestre pode ser fatal, tanto para a ocorrência de malformações congênitas estruturais no feto, quanto para problemas renais. Em se tratando da Mãe percebe-se que os AINEs podem prolongar seu tempo de parto, causar hemorragias e enfraquecer seus óvulos para uma futura reprodução.
\end{abstract}

Palavras-chave: Automedicação; Anti-inflamatórios não esteroides; Fetos e gestantes.

\begin{abstract}
Objective: To analyze the potential risks to pregnant women and fetuses caused by self-medication of non-steroidal anti-inflammatory drugs. Methods: The proposed methodology for this research follows a strategy of literature review, exploratory and descriptive research, whose collection was carried out in different databases, such as Scientific Electronic Library Online (SCIELO), records from both the Virtual Health Library (BVS), and records of the Medical Literature Analysis and Retrievel System Online (MEDLINE/PUBMED). The search took place through the use of the descriptors "SELF MEDICATION"; "PREGNANT", "AINES" and "RISKS". Results: It was noticed that the use of medication during pregnancy through the practice of self-medication with Ibuprofen 9 (27\%), Dipyrone $6(18 \%)$, Bromopride 2 (6\%), Paracetamol $13(40 \%)$, Dimenhydrinate $3(9 \%)$, being $31(94 \%)$ in the form of tablets was reported by 27 (33.75\%) pregnant women, and $3(11.1 \%)$ who had complaints of use such as headache, emesis and nausea and the indication was reported by $2(6 \%)$ pregnant by the mother and the others on their own. Conclusion: After analyzing several scientific articles, what is very clear is that the use of non-steroidal antiinflammatory drugs in early pregnancy has not shown to be a risk factor, however, after the first trimester it can be fatal, both for the occurrence of congenital structural malformations in the fetus, as for kidney problems. When it comes to the mother, it is clear that NSAIDs can prolong her delivery time, cause hemorrhages and weaken her eggs for future reproduction.
\end{abstract}

Keywords: Self-medication; Non-steroidal anti-inflammatory drugs; Fetuses and pregnant women. 


\begin{abstract}
Resumen
Objetivo: Analizar los riesgos potenciales para la gestante y el feto provocados por la automedicación de antiinflamatorios no esteroideos. Métodos: La metodología propuesta para esta investigación sigue una estrategia de revisión de la literatura, investigación exploratoria y descriptiva, cuya recolección se realizó en diferentes bases de datos, como la Biblioteca Electrónica Científica en Línea (SCIELO), registros tanto de la Biblioteca Virtual en Salud (BVS), y registros del Sistema de análisis y recuperación de literatura médica en línea (MEDLINE / PUBMED). La búsqueda se realizó mediante el uso de los descriptores "AUTOMEDICACIÓN"; "EMBARAZADA", "AINES" y "RIESGOS". Resultados: Se notó que el uso de medicación durante el embarazo mediante la práctica de la automedicación con Ibuprofeno 9 (27\%), Dipirona 6 (18\%), Bromoprida 2 (6\%), Paracetamol 13 (40\%), Dimenhidrinato $3(9 \%)$, siendo $31(94 \%)$ en forma de comprimidos fue reportado por $27(33,75 \%)$ mujeres embarazadas, y $3(11,1 \%)$ que tenían quejas de uso como dolor de cabeza, vómitos y náuseas y se informó la indicación en $2(6 \%)$ embarazadas por la madre y las demás solas. Conclusión: Luego de analizar varios artículos científicos, lo que está muy claro es que el uso de antiinflamatorios no esteroides en el inicio del embarazo no ha demostrado ser un factor de riesgo, sin embargo, luego del primer trimestre puede ser fatal, tanto para el aparición de malformaciones estructurales congénitas en el feto, como por problemas renales. Cuando se trata de la madre, está claro que los AINE pueden prolongar su tiempo de parto, causar hemorragias y debilitar sus óvulos para una futura reproducción.
\end{abstract}

Palabras clave: Automedicación; Fármacos anti-inflamatorios no esteroideos; Fetos y mujeres embarazadas.

\title{
1. Introdução
}

A automedicação consiste em uma prática do uso de medicamentos sem prescrição médica, no qual apresenta um fator de risco para a população, inclusive em gestantes que buscam utilizar práticas terapêuticas por conta própria, o uso do fármaco torna-se um fator principal com a finalidade de manter em equilíbrio a saúde, promovendo o alivio e a cura dos sintomas (Schimitt Júnior et al., 2013).

Nesse sentido, a fim de melhor orientar a paciente foi criada uma classificaçãode riscos para o uso de medicamentos durante a gestação, sendo uma das classificações mais conhecidas no mundo, utilizada desde 1979, é a categorização por letras do FDA (US Food and Drug Administration), que organiza os medicamentos em cincocategorias (A, B, C, D e X), sendo que a categoria D evidência positivamente risco fetal, mas os benefícios podem superar os riscos. Já a categoria X oferece risco fetal na gestação considerada como medicamento potencialmente perigoso de uso ambulatorial (Instituto para práticas Seguras no Uso de Medicamentos - ISPM, 2019).

Para Carvalho (2013), a classificação de riscos para o uso de medicamentos é necessária, porém deve se levar em consideração alguns fatores tais como: as doses administradas, a duração do tratamento, a via de exposição e o tempo gestacional, haja vista serem fatores que desencadeiam erros clínicos no momento em que são prescritos. Diante disto, no ano de 2014 a FDA passou a exigir especificações e atualizações de informações constantes em bula, não somente sobre o risco de malformação fetal, mas também sobre os impactos durante o parto, tais como: prolongamento do tempo de parto, risco de baixo peso ao nascer, risco de morte neonatal, impacto no crescimento e desenvolvimento da criança.

Os anti-inflamatórios (AINEs) quando administrados em mulheres grávidas dependendo do tipo, dose e a duração da terapia, o período gestacional e do tempo decorrido entre a administração e o parto, os níveis maternos destes medicamentos atravessam a placenta e podem causar efeitos adversos a mãe e ao feto, tais como: hipertensão, osteopenia, osteonecrose, maior risco de diabetes gestacional e a maior propensão a infecções. Vale ressaltar que, as gestantes possuem alterações em seu quadro fisiológico durante o período gestacional, acarretando diversas dores e mudanças. Quando se aproxima do parto, na tentativa de diminuir as dores de contrações as mesmas utilizam AINEs (Källén \& Reis, 2015; Antonucci et al., 2012).

Este trajeto que a medicação e/ou os fármacos fazem no corpo da gestante, chegam ao feto especialmente pela placenta, fazendo o mesmo percurso que o oxigênio, bem como os nutrientes necessários para o desenvolvimento do bebê perpassam. Mesmo não atravessando a placenta tais fármacos podem causar diferentes impactos no útero da gestante que 
consequentemente afetará o feto (Gunatilaki \& Patil, 2021).

Mesmo com tantas informaç̃es, Quintanilha (2020) e a FDA (2019), alertam quanto a automedicação, especialmente no que se refere aos AINEs, pois em estudos realizados descobriram que os mesmos comprometem a produção do líquido amniótico pelos rins dos bebês, que a partir da vigésima semana de gestação são seus principais produtores.

É de conhecimento que a gravidez não é doença, mas é um período que exige atenção e inspira cuidados. Seguindo essa vertente, Nascimento et al. (2016), afirma o quanto a medicação e a medicalização expõe tanto a mãe quanto o bebê a riscos, isto porque, muitas consomem medicamentos sem prescrição e/ou sem necessidade. Outras de fato precisam, depende muito das necessidades individuais das mesmas. Porém, seja para uso de suplementação nutricional ou para intervir em caso de intercorrências obstétricas deve-se analisar os ricos que os fármacos podem causar.

Albuquerque \& Tavares (2011), esclarece que podem surgir diferentes efeitos dependendo do fármaco utilizado. Estes efeitos recaem tanto nas mães quanto nos fetos e dependem da época de exposição durante a gestação, da frequência e da dose total, ocasionando potencialmente alterações na estrutura ou função do concepto e trazendo consequências farmacológicas e toxicológicas diversas.

Algo bem preocupante a ser citado, que foi objeto de estudo de Lunardi et al. (2014), é que mesmo que grande parte das mulheres gestantes façam uso de medicamentos no Brasil, as mesmas têm uma limitação no acesso a informações sobre segurança, isto porque a uma imensa dificuldade na obtenção de dados referentes ao uso de medicamentos em gestantes durante os ensaios clínicos randomizados. Outro fator vem a ser os dados de segurança às gestantes, que só são adquiridos através das técnicas observacionais e/ou notificações da Farmacovigilância que só são disponíveis após a comercialização.

Albuquerque e Tavares (2011), destaca ainda que, os AINEs modificam a faixa de seu risco de acordo com o tempo de gestação, sendo classificado como (C) até o final do segundo trimestre e jamais deve ser utilizado no terceiro trimestre, pois transforma-se em um fator de risco e passa na tabela de classificação para o (D) exatamente por causarem constrição do ducto arterioso fetal e acarretar em hipertensão arterial pulmonar.

A Agência Nacional de Vigilância Sanitária (ANVISA), destaca que no Brasil, vários medicamentos do grupo AINEs são encontrados e adquiridos de forma fácil em drogarias e farmácias. Dentre eles o Naproxeno, Ibuprofeno e Cetoprofeno, por constarem na lista de Medicamentos Isentos de Prescrição (MIP) desde 2003, mesmo possuindo um grau tóxico elevado para quem os consome. (Brasil, 2011). Diante deste contexto surge a seguinte problemática: Quais os riscos que a automedicação dos Anti-Inflamatórios não Esteroides podem causar em Gestantes e Fetos?

Com intuito de responder a situação problema, o presente artigo tem como objetivo analisar os riscos potenciais às Gestantes e Fetos, causados pela automedicação de AINEs.

\section{Metodologia}

A proposta de metodologia para esta pesquisa segue uma estratégia de revisão de literatura, pesquisa exploratória e descritiva, cuja coleta fora realizada em diferentes bases de dados. Os dados foram coletados mediante a leitura exploratória dos artigos que foram baixados no formato PDF e consulta aos livros acadêmicos. Nos meses de fevereiro a setembro de 2021 foram analisados diversos trabalhos elaborados e publicados entre os anos de 2011 a 2020, a fim de abranger investigações atuais e possíveis alterações sobre o tema. Foram realizadas buscas através dos descritores "AUTOMEDICAÇÃO", "GESTANTES", "AINES" e "RISCO", buscando artigos científicos em português a respeito do tema, considerando a primeira palavra mais frequente.

Além do uso da Biblioteca Virtual em Saúde (BVS) para efetuar a pesquisa, onde a mesma disponibiliza um portfólio diversificado de artigos científicos, promovendo a pesquisa em várias fontes bibliográficas, realizou-se também pesquisas 
suplementares no localizador de registros da Medical Literature Analysis and Retrievel System Online (MEDLINE/PUBMED), Scientific Electronic Library Online (SCIELO) e Artigos Científicos, com o objetivo de confirmar a existência de resultados adicionais e validar a relevância da pesquisa. De um total de 45 artigos lidos, 31 foram selecionados. Foi perceptível ao longo das leituras e dos estudos o quanto os artigos aprofundaram-se em pesquisa de campo, exatamente para comprovar os riscos que os fármacos trazem para mulheres gestantes, bem como a seus fetos.

Os critérios de inclusão utilizados foram: artigos em língua portuguesa e inglesa publicados no período estabelecido entre 2011 a 2021, bem como, os que abordaram a automedicação em gestantes, especialmente de anti-inflamatórios não esteroidais, estando em consonância com o objetivo proposto, não sendo inseridos os que não tem relação com o tema abordado. Foram critérios de exclusão: estudos que não abordavam relação com a temática em questão e artigos que haviam feito o mesmo estudo. Foi critério de exclusão também a automedicação em populações e amostras que não fossem gestantes.

\section{Resultados}

Os resultados foram construídos a partir de estudos desenvolvidos por diferentes profissionais da área da saúde, dentre eles estão Santos et al. (2017), que, constatou em Fortaleza, na Policlínica Francisco Carlos Cavalcante Roque, o quanto a prática de automedicação vinha sendo executada por elas. De um universo de 100 gestantes 33,75, ou seja, 27 se automedicavam e desse total 3 afirmaram sentir-se mal ao executar tal pratica.

Outro dado que os autores constataram foi que esse "sentir-se" mal era frequente ao utilizarem os medicamentos: Dipirona, Ibuprofeno e Dimenidrinato. Santos et al., (2017) foram mais além e pesquisaram quais medicamentos utilizados na automedicação e destacaram trinta e três, sendo: Ibuprofeno 9 (27\%), Dipirona 6 (18\%), Bromoprida 2 (6\%), Paracetamol 13 (40\%) e Dimenidrinato 3 (9\%). Desse total 31 (94\%) em forma de comprimidos, apresentando como queixas de uso a cefaleia, êmese e náuseas e a indicação foi relatada por 2 (6\%) gestantes pela a mãe e as demais por conta própria.

É notório a ampla utilização dos AINES na pesquisa anteriormente citada, não é à toa que Sanarmed (2019), frisou o quantos os AINEs constituem uma das classes de fármacos mais distribuídas pelo mundo, utilizados em tratamentos de dor aguda e crônica decorrente de processo inflamatório.

Não obstante, Santos et al. (2017), identificaram também que, dentre os medicamentos mais prescritos estão o Metildopa (20\%), Sulfato ferroso (11\%) e Cefalexina (8\%). Em se tratando de classificação terapêutica os autores frisaram que os Antianêmicos foram os campeões perfazendo um total de 20 gestantes respondentes como usuárias dos mesmos, o equivalente a 20,2\%. Em seguida vieram os Anti-hipertensivos, perfazendo um total de 19,1\%, os AINEs ocuparam o terceiro lugar, com um quantitativo bastante expressivo em relação ao demais (14,1\%), seguido de Antibiótico 13 (13,1\%), antiasmático $10(10,1 \%)$, antifúngico $4(4,1 \%)$, antidepressivo $4(4,1 \%)$, antiemético $4(4,1 \%)$, anticoagulantes $4(4,1 \%)$, antiviral $2(2 \%)$, antifibrinolítico anti-hemorrágico 2 (2\%), imunossupressores 1 (1\%), antiespasmódicos $1(1 \%)$ e antidiabético oral $1(1 \%)$.

Os AINES mais uma vez aparecem com representatividade no estudo, e é válido ressaltar que esses anti-inflamatórios se formam através de uma extensa classe de compostos heterogêneos com estruturas químicas variantes, podendo ser distribuídos em classes de acordo com seu grupo químico. Admite-se que as diferenças na ação primária entre fármacos estejam na distribuição em subclasses de acordo com sua seletividade (Carvalho \& Araújo, 2013). São classificados em inibidores não seletivos e inibidores seletivos da COX (Quadro 1) (Varalda \& Motta, 2011). 
Quadro 1. Como os AINES são classificados.

\begin{tabular}{|c|c|}
\hline Não seletivo & Seletivos da COX-2 \\
\hline Ácido Acetilsalicílico, & Rofecoxib \\
Ibuprofeno, & Celecoxib \\
Cetoprofeno, & Etoricoxib \\
Naproxeno, & \\
Piroxicam, & \\
Nimesulida, & \\
Meloxicam, & \\
Tenoxicam & \\
Diclofenaco de Sódio & \\
\hline
\end{tabular}

Fonte: Varanda e Motta (2011).

Os tradicionais AINEs inibem as mesmas enzimas de forma reversível e não- seletiva, no entanto, existem fármacos que acetilam as isoenzimas (COX-1 e COX-2) de forma irreversível. Sabe-se que as ações anti-inflamatória, antipirética e analgésica decorrem da inibição sobre a COX-2, enquanto os efeitos indesejáveis são resultantes da inibição da COX-1 (Silva et al., 2013).

No que tange as prescrições e levando em consideração os riscos dos medicamentos prescritos para as gestantes, em categoria C estavam o Lorazepam, Dipropionato de beclometasona, salbutamol e Escopolamina associado a Dipirona. E em classificação D, apresentaram o Ibuprofeno, de acordo com os dados expressos em quantidade de aparecimento nas prescrições, e mostrando que era no terceiro trimestre de gravidez que eles eram mais prescritos (Santos et al., 2017).

Apesar de ser geralmente bem tolerado, o consumo de Ibuprofeno pode desencadear dor de cabeça e de estômago, vômitos, diarreia, podendo também produzir diversas gastropatias, entre as quais, úlceras no estômago e duodeno, pois bloqueia a produção da barreira de proteção da mucosa gástrica (Seabra, 2015).

Ao analisar um estudo de Pinheiro e Wannmacher (2014), constatou-se que a associação entre uso de ácido acetilsalicílico e AINE pode ocasionar malformações congênitas. Nenhum medicamento associou-se significativamente à elevação de risco de malformação cardíaca. Porém, evidenciou- se associação significativa de risco de malformação orofacial quando o AINE foi usado durante o primeiro trimestre de gravidez (Berard, Zhao \& Sheehy, 2017).

No artigo de Schaefer et al., (2012), foram demonstrado que o fechamento prematuro do ducto arterial, evento raro em decorrência do uso de AINEs ocorre antes da $29^{\circ}$ semana, e é aumentado em $50-70 \%$ na $34^{\circ}$ semana, chegando a $100 \%$ a partir da $36^{\circ}$ semana de gravidez.

Os autores Li, Liu e Odouli (2003), ao fazer um estudo de base populacional demonstrou aumento de $80 \%$ na taxa de risco de aborto espontâneo associado a AINEs (HR =1,8; IC95\%: 1,0-3,2), aumentando esse risco com uso próximo da concepção $(H R=5,6$; IC95\%: 2,3-13,7) ou por mais de uma semana (HR=8,1; IC95\%: 2,8-23,4). Já no estudo de Ofori (2006), através de técnica observacional, o mesmo encontrou razão de chances ajustada de 2,21 (IC95\%: 1,71-2,85) para defeitos congênitos quando AINEs foram usados no primeiro trimestre de gravidez. Ainda apresentou OR de 3,34 (IC95\%: 1,87-5.98) para alterações relacionadas ao fechamento do septo cardíaco.

Um artigo de revisão sistemática que incluiu 22 estudos de casos e controles, sete cortes e um ensaio clínico aleatório investigou a associação entre uso de ácido acetilsalicílico e AINEs e risco de malformações congênitas (Nakhai-Pour \& Berard, 2011). Em relação a ácido acetilsalicílico (11 estudos), somente dois estudos de casos e controles demonstraram aumento significativo no risco de malformação (OR =1,64; IC95\%: 1,30-2,04).

Com respeito a uso de AINEs, dois estudos os associaram a aumento daquele risco. Nessa revisão, nenhum medicamento associou-se significativamente à elevação de risco de malformação cardíaca. Porém, dois estudos evidenciaram 
associação significativa de risco de malformação orofacial quando o AINE foi usado durante o primeiro trimestre de gravidez (Nakhai-Pour \& Berard, 2011).

\section{Discussão}

É evidente que o uso de AINE na Gravidez precisa ser moderado e requer atenção aos riscos. É necessário atentar ao tempo de gestação e mais as associações que serão feitas ao AINE. Na maioria dos estudos, o Ibuprofeno apresentou o maior uso no grupo de caso (4,46\%), controle $(0 \%)$ com $\mathrm{OR}=7,904$ e o valor de p borderline de $\mathrm{p}=0,05$. O termo "borderline", que em inglês significa "fronteiriço", teve origem na psicanálise: esses pacientes não podiam ser classificados como neuróticos (ansiosos e exagerados), nem como psicóticos (que enxergam a realidade de forma distorcida), mas estariam em um estado intermediário entre esses dois espectros (Associação Brasileira de Psiquiatria - ABP, 2018).

Estudos com culturas organotípicas de ovários humanos, demonstraram que o Ibuprofeno usado no início da gestação, pode ser deletéria para as células germinativas do ovário (Leverrier-Penna et al., 2018). Em estudos envolvendo ratos, o Ibuprofeno, a aspirina e o acetaminofeno obtiveram relação com a ocorrência da criptorquidia em filhos (Romani et al., 2014; Hurtado-Gonzalez et al., 2018).

"Não é só o Ibuprofeno, mas a maioria dos anti-inflamatórios não hormonais é contraindicado na gestação, porque há uma série de efeitos que podem surgir. Em casos extremos, no final da gestação, eventualmente é possível, mas nas primeiras 24 semanas de gestação é importante evitar ao máximo", explica Francisco Furtado Filho, médico ginecologista especialista em reprodução humana e diretor da clínica Fertway (Milléo, 2018).

"Se a gestante reclama de dor de cabeça no início da gestação, essa dor pode estar relacionada aos efeitos hormonais próprios do momento, a uma baixa de pressão, que também é fisiológica, ou eventualmente a uma hipoglicemia pela dificuldade de se alimentar, devido às náuseas e vômitos, e não a uma enxaqueca mesmo", reforça Francisco Furtado Filho, médico ginecologista (Milléo, 2018).

Apesar de evidenciar muitos riscos a gestantes e fetos, alguns estudos americanos recentes que tornaram complexos os entendimentos acerca dos AINEs, isto porque Lind JN et al., (2017), analisaram um estudo envolvendo mais 65.000 mulheres e demonstraram que o uso de AINES não é um fator de risco independente para abortamento. Ao fazer estudos em humanos sugerem associação entre o uso de AINES e redução da fertilidade feminina, sendo prudente evitar o seu uso em mulheres que estão tentando engravidar.

Em relação às malformações congênitas, a situação dos AINES é mais complexa. Na maioria dos estudos, o risco para qualquer malformação não apresenta aumento significativo com o seu uso, mas pode estar aumentado em algumas condições, notavelmente defeitos cardíacos. Por outro lado, um estudo que avaliou especificamente o risco de defeitos do septo interventricular não achou qualquer associação (Lind Jn et al., 2017).

A utilização de AINES no terceiro trimestre da gestação na mãe, pode estar relacionada ao trabalho de parto prolongado, hemorragia pós-parto, embora a administração por curto período dificilmente esteja associada ao fechamento do ducto arterial fetal. É comum a prática de evitar-se o uso de AINES após o período que se estende entre 28 e 32 semanas até o final da gestação (Bloor \& Paech, 2013).

Quanto aos inibidores seletivos da COX-2, esperava-se que apresentassem menos efeitos adversos que os não seletivos, mas os mesmos problemas estão presentes, como oligodrâmnio e fechamento prematuro do ducto arterial. Como há poucos trabalhos sobre o uso dessa classe de fármacos durante a gestação, eles são considerados classe $\mathrm{C}$ até o segundo trimestre, e D no terceiro trimestre (Wilmer, 2016). 


\section{Conclusão}

Após a análise dos artigos científicos, o que fica bem explicito é que, o uso de anti-inflamatórios não esteroidais no início da gravidez não demonstrou ser um fator de risco, porém, após o primeiro trimestre pode ser fatal, tanto para a ocorrência de malformações congênitas estruturais no feto, quanto para problemas renais. Em se tratando da Mãe percebe-se que os AINEs podem prolongar seu tempo de parto, causar hemorragias e enfraquecer seus óvulos para uma futura reprodução. Ao usar a terapia farmacológica deve se pedir orientação, passar por uma avaliação de médicos e farmacêuticos especializados, a fim de que sejam mostrados os riscos e benefícios para a mãe e para o feto, tendo-se o cuidado de oferecer todas as opções terapêuticas para garantir o bem-estar da gestante, minimizar a teratogenicidade fetal e evitar sintomas crônicos e incapacidade a longo prazo.

Vale frisar que, pouco se tem no Brasil estudos e pesquisas feitos em Humanos sobre os AINEs, tornando difícil o trabalho da ANVISA bem como de demais órgãos responsáveis pela indústria farmacêutica no Brasil. Muito se recorreu a estudos científicos realizados fora do país, haja visto como dito anteriormente nosso país ser carente de informação, estudos e pesquisas acerca do tema o que facilita a criação de Leis que facilitam o acesso desse medicamento por parte de um percentual elevado da população.

Neste sentido fica como sugestão para futuros pesquisadores realizarem mais estudos clínicos randomizados em Gestantes, a fim de fazer com que a pesquisa se torne mais assertiva, consequentemente contribuindo para minimizar os riscos inerentes à terapia medicamentosa, sem deixar de lado é obvio a segurança dos mesmos, buscando sempre atuar em um período de Gestação que não venha causar-lhes mal algum.

Outros estudos práticos podem ser realizados em pacientes grávidas que possuem doenças crônicas e são obrigadas a ingerir determinados medicamentos, sem escolhas e, mesmo tendo ocorrido um avanço tecnológico, Justina et al., (2018) relatam que 5\% a 10\% das malformações são induzidas por medicamentos e essas malformações estão entre as dez principais causas de mortalidade infantil no mundo.

Muitas mulheres se automedicam sem ao menos ter conhecimentos dos possíveis riscos e males que podem causar a si e consequentemente ao seu feto. Se faz necessário urgentemente uma reformulação nas diretrizes Nacionais para que muitos medicamentos, inclusive os anti-inflamatórios não esteroidais sejam obrigatoriamente prescritos e comprados mediante receituário válido.

\section{Referências}

Albuquerque, V. M. T. \& Tavares, C. A. (2011). Avaliação de indicadores de medicamentos: importância para a qualidade na prescrição médica. R. Bras. Farm. Hosp. Serv. Saúde São Paulo, 2(3), 31-35.

Antonucci, R., Zaffanello, M., Puxeddu, E., Porcella, A., Cuzzolin, L., Pilloni, M. D. \& Fanos, V. (2012). Use of Non-steroidal Anti-inflammatory Drugs in Pregnancy: Impact on theFetus and Newborn. Curr Drug Metab, 13(4), 474-90. DOI: 10.2174 / 138920012800166607

Bérard, A., Zhao, J-P. \& Sheehy, O. (2017). Antidepressant use during pregnancy and the risk of major congenital malformations in a cohort of depressed pregnant women: an updated analysis of the Quebec pregnancy cohort. BMJ Open, 7(1), 1-13.

Bloor, M. \& Paech, M. (2013). Nonsteroidal anti-inflammatory drugs during pregnancy and the initiation of lactation. Anesth Analg. 116(5), $1063-1075$.

Brasil. Associação Brasileira de Psiquiatria. (2018). Transtorno de Personalidade. https://www.abp.org.br/post/abptv-transtornos-de-personalidade

Brasil. Agência Nacional de Vigilância Sanitária (Anvisa). (2011). Resolução RDC nº 138, de 29 de maio de 2003. Dispõe sobre o enquadramento na categoria de venda de medicamentos. Diário Oficial da União, Poder Executivo, Brasília, DF.

Carvalho, V. C. P. \& Araújo, T. V. B. (2013). Adequação da assistência pré-natal em gestantes atendidas em dois hospitais de referência para gravidez de alto risco do Sistema Único de Saúde, na cidade de Recife, Estado de Pernambuco. Rev. Bras. Saúde Matern.Infant, 7(3), $309-317$.

FDA. Food and Drug Administration. (2019). Content and Format of Labeling for Human Prescription Drug and Biological Products; Requirements for Pregnancy and LactationLabeling [Internet]. https://s3.amazonaws. com/public-inspection.federalregister.gov/2014-28241.pd 
Gunatilake, R., \& Patil, S. (2021). Uso de medicamentos durante a gravidez - Problemas de saúde feminina. Manual MSD Versão Saúde para a Família. https://www.msdmanuals.com/pt-br/casa/problemas-de-sa\%C3\%BAde-feminina/uso-de-medicamentos-drogas-durante-a-gesta\%C3\%A7\%C3\%A3o

Hurtado-Gonzales, P., Anderson, R. A., Macdonald, J., Driesche, S. V. D., Kilcoyne, K., Jorgenses, A., Mckinnel, C., Macpherson, S., Sharpe, R. \& Mitchell, R. (2018). Effects of exposure to acetaminophen and ibuprofen of fetal germ cell development in both sexes in rodent and human using multiple experimental systems. Environ Health Perspect, 16(126), 1 - 17.

ISMP. Instituto para Práticas Seguras no Uso de Medicamentos. (2020). Desafio global de segurançado paciente: medicação sem danos [Internet]. Boletim ISMP Brasil. 7(1), 1-8.

Kallén, B. \& Reis, M. (2015). Useof tramadol in early pregnancy and congenital malformation risk. Reproductive Toxicology, 58, $246-251$.

Leverrier-Penna, S., Mitchell, R. T., Becker, E., Lecante, L., Maamar, M.B., Homer, N., Lavoué, V., Kristense, D. M., Dejucq-rainsford, N. \& Jécou, B. (2018). Ibuprofen is deleterious for the development of first trimester human fetal ovary ex vivo. Human Reproduction, 33(3), 482-493.

Li, D. K., Liu, L. \& Odouli, R. (2003). Exposure to non-steroidal anti-inflammatory drugs during pregnancy and risk of miscarriage: population based cohort study. BMJ, 327(7411), 368-372.

Lind, J. N., Interrante, J. D., Ailes, E. C., Gilboa, S. M., Khan, S., Frey, M. T., Dawson, A. L., Honein, M. A., Dowling, N. F., Razzaghi, H., Creanga, A. A. \& Broussard, C. S. (2017). Maternal use of opioids during pregnancy and congenital malformations: a systematic review. Pediatrics. 139(6), 1-35.

Lunardi-Maia, T., Schuelter-TrevisoL, F. \& Galato, D. (2014). Uso de medicamentos no primeiro trimestre de gravidez: avaliação da segurança dos medicamentos e uso de ácido fólico e sulfato ferroso. Rev Bras Ginecol Obstet, 36(12), 541-547.

Milléo, A. (2018). Ibuprofeno usado na gravidez prejudica a fertilidade das futuras gerações, aponta estudo. https://www.gazetadopovo.com.br/viverbem/saude-e-bem-estar/anti-inflamatorio-na-gestacao-pode-prejudicar-fertilidade

Nakhai-Pour, H. R. \& Berard, A. (2011). Major malformations after first-trimester exposure to aspirin and NSAIDs. Expert. Rev. Clin. Pharmacol., 1(5), 605616.

Nascimento, A. M., Gonçalves, R. E. L. M., Medeiros, R. M. K. \& Lisboa, H. C. F. (2016). Avaliação do uso de medicamentos por gestantes em Unidades Básicas de Saúde de Rondonópolis, Mato Grosso. Revista Eletrônica Gestão \& Saúde, 07(01), 96-113.

Ofori, B., Oraichi, D., Blais, L., Rey, E. \& Bérard, A. (2006). Risk of congenital anomalies in pregnant users of non-steroidal anti-inflammatory drugs: a nested case-control study. Birth Defects Res. B. Dev. Reprod. Toxicol., 77(4), 268-279.

Pinheiro, R. M. \& Wannmacher, L. (2014). Uso racional de anti-inflamatórios não J. M. Silva, et al. ISSN 1983-6708 Revista Científica do ITPAC, 7(4), Pub.5, esteroides. In: Ministério Da Saúde. Uso Racional de Medicamentos Temas relacionados. Brasília: Editora MS, 2012. Cap. 5, p. 41-50

Quintanilha, D. O. (2020). Agência Americana orienta evitar anti-inflamatórios na gravidez. https://pebmed.com.br/agencia-americana-orienta-evitar-antiinflamatorios-na-gravidez

Romani, F., Tropea, A., Scarinci, E., Federico, A., Dello Russo, C., Lisi, L., Catino, S., Lanzone, A., \& Apa, R. (2014). Endocrine disrupters and human reproductive failure: the in vitro effect of phthalates on human luteal cells. Fertil Steril, 102(3), 831-837.

Sanamed. (2019). Anti-inflamatórios Não Esteroides (AINES): o que são e como funcionam. Https://www.sanarmed.com/anti-inflamatorios-nao-esteroidesaines.

Santos, S., Alves, H. \& Barros, K. (2017). Estudo de indicadores de prescrição em gestantes de alto risco de um serviço de referência. Revista Brasileira de Farmácia Hospitalar e Serviços de Saúde, 8(4)25-30.

Seabra, C. I. R. (2015). Farmacocinética do Ibuprofeno. Tese de Doutorado.

Schaefer, C., Peters, P. W. \& Miller, R. K. (2014). Drugs during pregnancy and lactation: treatment options and risk assessment. Academic Press.

Schmitt Júnior, A.A. Lindner, S. \& De Santa Helena, E.T. (2013). Avaliação da adesão terapêutica em idosos atendidos na atenção primária. Rev Ass Med Bras, 59(6), 614-621.

Silva, J. A. C. da, Gomes, A. L., Oliveira, J. P. S. de, Sassaki, Y. de A., Maia, B. T. B. \& Abreu, B. M. de. (2013). Prevalência de automedicação e os fatores associados entre os usuários de um Centro de Saúde Universitário. Rev Bras Clin Med, 11(1), 27-30

Varalda, D. B. \& Motta, A. A. (2011). Reações adversas aos antiinflamatórios não esteroidais. Rev. bras. alergia imunopatol, 32(1), 27-34.

Wilmer, E., Chai, S. \& Kroumpouzos, G. (2016). Drug safety: pregnancy rating classifications and controversies. Clin Dermatol, 34(3), 401-409. 